\title{
STUDY ON ESSENTIAL OIL COMPOSITION OF THE ROOTS OF CRASSOCEPHALUM CREPIDIOIDES (BENTH.) S. MOORE
}

\author{
RAJESH K. JOSHI* \\ Department of Phytochemistry, Regional Medical Research Centre (Indian Council of Medical Research), \\ Belgaum, Karnataka-590 010, India \\ (Received: August 14, 2013 - Accepted: January 24, 2014)
}

\begin{abstract}
The aim of the present study was to analyzed chemical constituents of the essential oil, obtained by hydro distillation of the roots of Crassocephalum crepidioides (Benth.) S. Moore. The oil was analyzed using gas chromatography equipped with flame ionization detector (GC-FID) and gas chromatography coupled with mass spectrometry (GC/MS). Forty-seven compounds were identified, representing $93.2 \%$ of the total oil. The main constituents were $(E)$ - $\beta$-farnesene (30.6\%), $\alpha$-humulene $(10.3 \%), \beta$-caryophyllene $(7.2 \%)$, cis- $\beta$-guaiene $(6.1 \%)$ and $\alpha$-bulnesene $(5.3 \%)$. The oil was constituted mainly of sesquiterpene hydrocarbons (77.6\%), followed by oxygenated sesquiterpenes $(8.7 \%)$, oxygenated monoterpenes $(3.5 \%)$, monoterpene hydrocarbons $(2.8 \%)$ and phenyl derivatives $(0.6 \%)$. This is the first report on the essential oil composition of the roots of $C$. crepidioides.
\end{abstract}

Keywords: Crassocephalum crepidioides (Benth.) S. Moore, Essential oil composition, (E)- $\beta$-Farnesene, GC/MS.

\section{INTRODUCTION}

Crassocephalum crepidioides (Benth.) S. Moore (syn. Gynura crepidioides Benth.) commonly known as Thickhead or Fireweed is belongs to the family Asteraceae. The genus Crassocephalum is represented approximately by 100 species distributed in Asia, Africa, Australia, Malaysia, China, Nepal and Sri Lanka. In India, the genus Crassocephalum is represented by 8 species [1]. This plant is used as traditional medicine for treatment of cut wound and to cure diarrhea in various countries like India, Nepal and China [2-6]. It is a medicinal plant having free radical scavenging and hepatoprotective actions [7], antioxidant activity [8], and lipid peroxidation inhibitory effect [9]. There are few literature reports on the phytochemical analysis of this plant. Jacobine and jacoline very toxic pyrrolizidine alkaloids have been isolated from aerial parts of this species [10]. The essential oil of the flowers of the $C$. crepidioides has been reported from India to possess myrcene, $\beta$-phellandrene, dauca-5,8-diene, trans- $\beta$-farnesene and daucene as the major constituents, while the leaf oil was found to contain myrcene, $\beta$-phellandrene, allo-aromadendrene, $\alpha$-muurolene and $\beta$-panasinsene as the major components [11]. Leaves oil of $C$. crepidioides from Cameroon were $\alpha$-phellandrene, $p$-cymene, myrcene, limonene and $(E)$ $\beta$-ocimene as the major constituents [12]. In another report from Nigeria, the leaf oil contained $\alpha$-caryophyllene, $\beta$-cubebene and $\alpha$-farnesene, while thymol, $\alpha$-caryophyllene and 4-cyclohexybutyramide were found as the major constituents from stems oil of C. crepidioides [13]. To the best of author's knowledge, this is the first report on the chemical composition of the essential oil from the roots of C. crepidioides.

\section{EXPERIMENTAL}

\section{Plant Material}

The roots of $C$. crepidioides were collected in the month of September, 2011 from district Belgaum (Latitude N 15.88668; Longitude E 74.52353), Karnataka, India, at a height of $800 \mathrm{~m}$. The plant herbarium and voucher specimen (RMRC-582) has been deposited in Regional Medical Research Centre (RMRC) Belgaum, Karnataka, India.

\section{Isolation of Essential Oil}

The fresh plant material $(250 \mathrm{~g})$ was subjected to hydro-distillation using Clevenger type apparatus for $3 \mathrm{~h}$. The oil was collected and dried over anhydrous sodium sulfate and stored in sealed vials at $-4^{\circ} \mathrm{C}$ until analysis. The oil yield was $0.03 \%(\mathrm{v} / \mathrm{w})$

\section{GC-FID and GC-MS Analysis of the Essential Oil}

The oil was analyzed by using a Varian 450 (TG-5, $30 \mathrm{~m} \times 0.25 \mathrm{~mm}$ i.d., $0.25 \mu \mathrm{m}$ film thickness) Gas Chromatograph under the experimental conditions reported earlier $[14,15]$. The oven temperature was programmed from $60-$ $220^{\circ} \mathrm{C}$ at $3^{\circ} \mathrm{C} / \mathrm{min}$, using nitrogen as carrier gas. The injector temperature was $230^{\circ} \mathrm{C}$ and the detector (FID) temperature $240^{\circ} \mathrm{C}$. GC-MS utilized a Thermo Scientific Trace Ultra GC interfaced with a Thermo Scientific ITQ 1100 Mass Spectrometer fitted with a TG-5 (Thermo Scientific) fused silica capillary column $(30 \mathrm{~m} \times 0.25 \mathrm{~mm} ; 0.25 \mu \mathrm{m}$ film thickness). The oven temperature range was $60-220^{\circ} \mathrm{C}$ at $3^{\circ} \mathrm{C} / \mathrm{min}$ using helium as carrier gas at $1.0 \mathrm{~mL} / \mathrm{min}$. The injector temperature was $230^{\circ} \mathrm{C}$, and the injection volume $0.1 \mu \mathrm{L}$ in $n$-hexane, with a split ratio of 1:50. MS were taken at $70 \mathrm{eV}$ with a mass range of $\mathrm{m} / \mathrm{z} 40$ 450. All the experimental parameters were applied from those reported earlier $[16,17]$.

\section{Identification of the Components}

Identification of constituents were done on the basis of Retention Index (RI, determined with reference to homologous series of $n$-alkanes $\mathrm{C}_{8}-\mathrm{C}_{25}$, under identical experimental condition), MS library search (NIST 08 MS Library and WILEY MS $9^{\text {th }}$ Edition), and by comparison with MS literature data [18]. The relative amounts of individual components were calculated based on GC peak area (FID response) without using correction factor.

\section{RESULTS AND DISCUSSION}

Forty-seven constituents were characterized and identified by GC-MS, comprising $93.2 \%$ of the total oil. The identified compounds are listed in Table 1 in elution order from the TG-5 column, along with the percentage composition of each component and its retention index. The major constituents of the oil were $(E)$ - $\beta$-farnesene $(30.6 \%)$ (Fig.1), $\alpha$-humulene $(10.3 \%)$, $\beta$-caryophyllene (7.2\%), cis- $\beta$-guaiene (6.1\%) and $\alpha$-bulnesene $(5.3 \%)$. The essential oil consists mainly of 18 sesquiterpene hydrocarbons ( $77.6 \%)$, followed by 12 oxygenated sesquiterpenes $(8.7 \%), 1$ oxygenated monoterpene $(3.5 \%)$, 14 monoterpene hydrocarbons $(2.8 \%)$ and 2 phenyl derivatives $(0.6 \%)$. The composition of the essential oil often changes between different plant parts [19]. The difference in the complex composition of essential oils of one kind may sometimes be difficult to assign to specific chemotypes. The formation of essential oils depends on the tissue differentiation (secretory cells and excretion cavities, etc.) and on ontogenetic phase of the respective plant [20]. The secondary volatile metabolites of different parts of same plant virtually showed qualitative and quantitative differences of compounds reported in the essential oil composition from aerial parts and flowers of Anaphalis contorta [21-23], Craniotome furcata [24,25], Chromolaena odorata [26], Coleus aromaticus [27], Tagetes minuta [28], Curcuma longs [29], Artemisia persica [30], Senecio belgaumensis [31,32], Prangos ferulacea [33] and Lantana camara [34] and sometime derived different chemotypes from the roots of same plant $[29,30,35]$. The conclusion of this study revealed that the roots of $C$. crepidioides produced different chemotypes, namely, $(E)$ - $\beta$-farnesene, $\alpha$-humulene, $\beta$-caryophyllene, cis- $\beta$-guaiene and $\alpha$-bulnesene, other than reported from the leaf, stem and flower parts oils (Table 2).

\section{ACKNOWLEDGMENTS}

The author is grateful to Indian Council of Medical Research, New Delhi, India for providing necessary facilities. Thanks also to Mr. Manjunath Patil, Lab Attendant for collection of plant material and extraction of oil. 


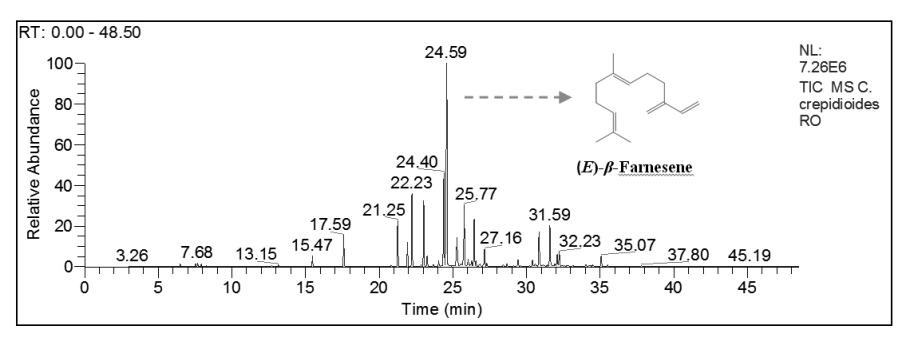

Fig1. GC-TIC chromatogram of the essential oil of the roots of C. crepidioides and its major compound $(E)-\beta$-farnesene.

Table 1. Percentage composition of essential oil of the roots of C. crepidioides

\begin{tabular}{|c|c|c|c|}
\hline Compound & RI & $\%$ & Identification* \\
\hline Sabinene & 979 & $\mathrm{t}$ & RI, MS \\
\hline$\beta$-Pinene & 984 & $\mathrm{t}$ & RI, MS \\
\hline Myrcene & 994 & 0.5 & RI, MS \\
\hline$\alpha$-Phellandrene & 1009 & $\mathrm{t}$ & RI, MS \\
\hline$p$-Cymene & 1030 & 0.2 & RI, MS \\
\hline$\beta$-Phellandrene & 1036 & 0.6 & RI, MS \\
\hline (Z)- $\beta$-Ocimene & 1045 & 0.4 & RI, MS \\
\hline (E)- $\beta$-Ocimene & 1056 & 0.2 & RI, MS \\
\hline$\gamma$-Terpinene & 1064 & $\mathrm{t}$ & RI, MS \\
\hline Terpinolene & 1093 & $\mathrm{t}$ & RI, MS \\
\hline Linalool & 1105 & 0.1 & RI, MS \\
\hline Borneol & 1170 & 0.3 & RI, MS \\
\hline Terpin-4-ol & 1183 & 0.4 & RI, MS \\
\hline$\alpha$-Terpineol & 1195 & 0.1 & RI, MS \\
\hline Thymol, methyl ether & 1242 & $\mathrm{t}$ & RI, MS \\
\hline Carvacrol, methyl ether & 1251 & 0.6 & RI, MS \\
\hline Bornyl acetate & 1293 & 3.5 & RI, MS \\
\hline Cyclosativene & 1379 & 0.2 & RI, MS \\
\hline$\alpha$-Copaene & 1383 & 3.5 & RI, MS \\
\hline Isocomene & 1392 & 0.2 & RI, MS \\
\hline$\beta$-Elemene & 1397 & 3.4 & RI, MS \\
\hline Cyperene & 1406 & 3.7 & RI, MS \\
\hline$\alpha$-Gurjunene & 1417 & $\mathrm{t}$ & RI, MS \\
\hline$\beta$-Caryophyllene & 1428 & 7.2 & RI, MS \\
\hline 2,5-Dimethoxy- $p$-cymene & 1433 & 0.5 & RI, MS \\
\hline trans- $\alpha$-Bergamotene & 1441 & 0.5 & RI, MS \\
\hline$\alpha$-Humulene & 1460 & 10.3 & RI, MS \\
\hline (E)- $\beta$-Farnesene & 1466 & 30.6 & RI, MS \\
\hline$\beta$-Chamigrene & 1484 & 3.0 & RI, MS \\
\hline cis- $\beta$-Guaiene & 1499 & 6.1 & RI, MS \\
\hline$\alpha$-Selinene & 1503 & 1.1 & RI, MS \\
\hline$\alpha$-Muurolene & 1506 & $\mathrm{t}$ & RI, MS \\
\hline$\alpha-B$ ulnesene & 1512 & 5.3 & RI, MS \\
\hline Cubebol & 1525 & 0.8 & RI, MS \\
\hline$\delta$-Cadinene & 1533 & 1.7 & RI, MS \\
\hline Occidentalol & 1557 & 0.2 & RI, MS \\
\hline Germacrene B & 1572 & 0.3 & RI, MS \\
\hline
\end{tabular}

\begin{tabular}{|c|c|c|c|}
\hline Ledol & 1577 & $\mathrm{t}$ & RI, MS \\
\hline Caryophyllene oxide & 1590 & 1.2 & RI, MS \\
\hline Guaiol & 1612 & 0.9 & RI, MS \\
\hline Hinesol & 1651 & 2.8 & RI, MS \\
\hline Valerianol & 1667 & 2.0 & RI, MS \\
\hline Occidentalol acetate & 1691 & $\mathrm{t}$ & RI, MS \\
\hline Cedr-8-en-13-ol & 1699 & $\mathrm{t}$ & RI, MS \\
\hline 14-oxy- $\alpha$-Muurolene & 1776 & 0.4 & RI, MS \\
\hline Cedrene-8,13-ol acetate & 1809 & 0.2 & RI, MS \\
\hline Khusinol acetate & 1831 & 0.2 & RI, MS \\
\hline \multicolumn{2}{|l|}{ Monoterpene hydrocarbons } & \multicolumn{2}{|c|}{2.8} \\
\hline \multicolumn{2}{|l|}{ Oxygenated monoterpenes } & \multicolumn{2}{|c|}{3.5} \\
\hline \multicolumn{2}{|c|}{ Sesquiterpene hydrocarbons } & \multicolumn{2}{|c|}{77.6} \\
\hline \multicolumn{2}{|c|}{ Oxygenated sesquiterpenes } & \multicolumn{2}{|c|}{8.7} \\
\hline \multicolumn{2}{|l|}{ Phenyl derivatives } & \multicolumn{2}{|c|}{0.6} \\
\hline \multicolumn{2}{|l|}{ Total identified } & \multicolumn{2}{|c|}{$93.2 \%$} \\
\hline
\end{tabular}

$\mathrm{RI}=$ Retention index relative to $\mathrm{C}_{8}-\mathrm{C}_{25} n$-alkanes on TG-5 column, MS= NIST and Wiley library and the literature, $\mathrm{t}=$ trace $(<0.1 \%)$.

Table 2. Comparative analysis between major components of essential oil of leaf, stem flower and root of $C$. crepidioides.

\begin{tabular}{|c|c|}
\hline Plant parts & Major compounds \\
\hline Leaf & $\begin{array}{l}\beta \text {-Phellandrene, allo-aromadendrene, } \alpha \text {-muurolene } \\
\beta \text {-panasinsene [11], } \alpha \text {-phellandrene, } p \text {-cymene, myrcene, } \\
\text { limonene, } \quad(E) \text { - } \beta \text {-ocimene [12], } \alpha \text {-caryophyllene, } \\
\beta \text {-cubebene and } \alpha \text {-farnesene [13] }\end{array}$ \\
\hline Stem & Thymol, $\alpha$-caryophyllene and 4-cyclohexybutyramide [13] \\
\hline Flower & $\begin{array}{l}\text { Myrcene, } \beta \text {-phellandrene, dauca-5,8-diene, trans- } \beta \text { - } \\
\text { farnesene and daucene [11] }\end{array}$ \\
\hline Root & $\begin{array}{l}\text { (E)- } \beta \text {-Farnesene, } \alpha \text {-humulene, } \beta \text {-caryophyllene, cis- } \beta \text { - } \\
\text { guaiene and } \alpha \text {-bulnesene }\end{array}$ \\
\hline
\end{tabular}

\section{REFERENCES}

1. P. K. Hajra, R. R. Rao, D. K. Singh, B. P. Uniyal Flora of India. BSI Publication, Kolkata, India, 1995.

2. S. R. Hynniewta, V. Kumar Indian J Tradit. Know. 7, 581, (2008).

3. K. S. Rajbhandari Ethnobotany of Nepal. Ethobotanical Society of Nepal C/o Central Department of Botany Kathmandu, Nepal, 2001.

4. N. P. Manandhar Plants and People of Nepal. Timber Press, Portland, Oregon, 2002.

5. S. R. Baral, P. P. Kurmi A Compendium of Medicinal Plants in Nepal. Mrs. Sharma R. 281 Maiju Bahal, Chabahill, Kathmandu-7, Nepal, 2006.

6. E. Roeder, Pharmazie 55, 711, (2000).

7. Y. Aniya, T. Koyama, C. Miyagi, M. Miyahira, C. Inomata, S. Kinoshita, T. Chiba, Biol. Pharm. Bull. 28, 19, (2005).

8. O. A. Odukoya, S. A. Inya-Agha, F. A. Segun, M. O. Sofidiya, O. O. Ilori Am. J. Food Tech. 2, 169, (2007).

9. S. O. Salawu, A. A. Akindahunsi, P. Cornuzzo, J. Pharmacol. Toxicol. 1, 429, (2006).

10. Y. Asada, M. Shiraishi, T. Takeuchi, Y. Osawa, T. Furuya, Planta Med. $51,539,(1985)$.

11. R. K. Joshi, Int. J Nat. Prod. Res. 1, 19, (2011).

12. P. H. A. Zollo, J. R. Kuiate, C. Menut, J. M. Bessiere, J. Essent Oil Res. $12,533,(2000)$.

13. I. A. Owokotomo, O. Ekundayo, I. A. Oladosu, S. A. Aboaba, Int. J. Chem. 4, 34, (2012).

14. R. K. Joshi, Nat. Prod. Commun. 8, 401, (2013).

15. R. K. Joshi, Natl. Acad. Sci. Lett. 36, 349, (2013).

16. R. K. Joshi, Nat. Prod. Commun. 6, 1145, (2011).

17. R. K. Joshi, J. Ethnopharmacol. 145, 621, (2013). 
18. R. P. Adams, Identification of essential oil components by gas chromatography/mass spectrometry, $4^{\text {th }}$ ed. Allured Publ. Corp., Carol Stream, IL, 2007.

19. C. B. Johnson, A. Kazantzis, M. Skoula, U. Mitteregger, J. Novak, Phytochem. Anal. 15, 286, (2004).

20. C. Franz, J Novak (2010) CRC Press, New York, USA, 2010

21. A. K. Sinha, M. S. Mehra, G. K. Sinha, Indian Perfum. 18, 35, (1974).

22. C. Pande, R. K. Joshi, S. S. Sammal, J. Essent. Oil Res. 20, 444, (2008).

23. R. K. Joshi, Nat. Prod. Commun. 8, 225, (2013).

24. R. K. Joshi, C. Pande, Nat. Prod. Commun. 3, 923. (2008).

25. R. K. Joshi, C. Pande, J. Essent. Oil Res. 21, 270, (2009).

26. R. K. Joshi, J. Essent. Oil Bear. P1. 16, 71, (2013).

27. R. K. Joshi, V. Badakar, S. D. Kholkute, Adv. Environ. Biol. 5, 1307,
(2011).

28. E. R. Chamorro, G. Ballerini, A. F. Sequeira, G. A. Velasco, M. F. Zalazar, J. Argent. Chem. Soc. 96, 80, (2008).

29. N. K. Leela, A. Tava, P. M. Shahj, S. P. John, B. Chempakam, Add Pharm. $52,137,(2002)$

30. B. F. Mirjalili, M. H. H. Meybody, M. M. Ardakani, A. Rustaiyan, N. Ameri, S. Masoudi, A. Bamoniri, J. Essent. Oil Res. 18, 544, (2006).

31. R. K. Joshi, Nat. Prod. Commun. 6, 1145, (2011).

32. R. K. Joshi, Chem. Nat. Compd. 47, 1010, (2012).

33. M. T. Akbari, A. Esmaeili, A. H. Zarea, N. Saad, F. Bagheri, Bulg. Chem. Commun. 42, 36, (2010).

34. R. K. Joshi, J. Biol. Act. Prod. Natr. 2, 135, (2012).

35. R. K. Joshi, J. Chem. 2013, 1, (2013). 